\title{
Letter to the Editor: Dengue fever in the Corpus Hippocraticum
}

\author{
Elias E Mazokopakis
}

Department of Internal Medicine, Naval Hospital of Crete, Chania, Greece

\begin{abstract}
Dengue fever (DF) is a globally significant infection which causes a range of severe and non-severe clinical manifestations. It is transmitted by mosquitoes of the Aedes genus (primarily Aedes aegypti, but also Aedes albopictus). In this letter, a possible DF epidemic on Thasos Island in Greece, which is described in the Book of Epidemics I of the Corpus Hippocraticum, is presented and analyzed. To my knowledge, it is the first report of DF in the history of medicine.

Keywords: Dengue fever, Corpus Hippocraticum.

DOI: https://dx.doi.org/10.4314/ahs.v20i3.19

Cite as: Marokopakis EE. Letter to the Editor: Dengue fever in the Corpus Hippocraticum. Afri Health Sci. 2020;20(3): 1166-1167. bttps:/ / dx.doi.org/10.4314/abs.v20i3.19
\end{abstract}

Dengue fever (DF) is a globally significant arboviral infection which causes a range of severe to non-severe clinical manifestations. ${ }^{1,2}$ It is transmitted by mosquitoes of the Aedes genus (primarily Aedes aegypti, but also $A e-$ des albopictus), an insect found in tropical and subtropical regions. ${ }^{1,2}$ The DF epidemic of 1927-1928 in Greece, which first affected the Athens area, was the most recent DF epidemic in Europe transmitted by Aedes aegypti mosquitoes. ${ }^{3}$ However, in my opinion, an epidemic of DF in Greece was probably described, centuries before, in the Corpus Hippocraticum, a compilation of works written by various authors throughout the $5^{\text {th }}$ and $4^{\text {th }}$ centuries BC and was subsequently associated with the renowned physician Hippocrates of Cos (c. 460-c. 370 $\mathrm{BC}$ ). To my knowledge, it is the first report of DF in the history of medicine. In particular, this DF epidemic is described in the Book of Epidemics I (third state) of the Corpus Hippocraticum. ${ }^{4}$ According to the author of the Book, after the intense storms that broke out in the March equinox, an epidemic of fever accompa-

\section{Corresponding author: \\ Elias E. Mazokopakis, \\ Naval Hospital of Crete, \\ Souda, Chania, 73 200, Crete, Greece. \\ Tel.: (+30) 2821082754 , \\ Fax: (+30) 2821082510 . \\ E-mail: emazokopakis@yahoo.gr}

nied by shivering and sweating, nose bleeds (epistaxis) and vaginal bleeding (in women of childbearing age), appeared in most of the residents of Thasos Island, Greece, from the beginning of the spring until the September equinox. Some of these patients had also jaundice. The febrile patients, who had epistaxis, also exhibited dysentery (hemorrhagic diarrhea) and at the same time acute renal failure (oliguria alternating with polyuria). The febrile patients who did not have epistaxis experienced "edema in the ears and weight sensation in the left flank to the top of the hip" (probably cervical lymphadenopathy and splenomegaly). Also, some of the febrile patients, mainly children, fell into lethargy, while pregnant women either miscarried or died. According to the author, the outcome of febrile patients was almost good. Based on the pre-mentioned clinical features and the outcome of patients, we can consider viral hemorrhagic fever as the most likely cause of the epidemic on Thasos Island. The exclusion of other diseases characterized by fever, hemorrhagic manifestations (due to thrombocytopenia), dysentery and renal insufficiency, such as leptospirosis (Weil's disease) and uremic hemolytic syndrome by enteropathogenic microbes (E. coli O157:H7, Shigella dysenteriae), was mainly based on the seasonal outbreak of the epidemic, the absence of jaundice in most patients, the simultaneous onset of dysentery with acute renal failure, the involvement of all ages and the good outcome for most patients. It is my firm belief that among the RNA viruses which have been recognized as being able to cause hem-

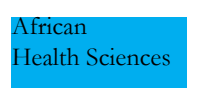

(C) 2020 Mazokopakis EE. Licensee African Health Sciences. This is an Open Access article distributed under the terms of the Creative commons Attribution License (https://creativecommons.org/licenses/BY/4.0), which permits unrestricted use, distribution, and reproduction in any medium, provided the original work is properly cited. 
orrhagic fevers in Greece, the most likely is the dengue virus belonging to the Flavivirus genus/Flaviviridae family. ${ }^{1,2}$ The patients in the Thasos Island epidemic probably experienced the main symptoms of either DF (two phases: febrile and convalescent) or dengue hemorrhagic fever/dengue shock syndrome (three phases (febrile, critical, and convalescent). ${ }^{1,2}$ Considering that ancient Thasos was a trading center with two harbors, famous for its wine and marbles in the known world at that time (Europe, Asia and Africa), and the spring was the starting point for commerce, it is possible that harbors were the entrance gates for the transmitter mosquito of DF. Moreover, the described weather conditions in the Book of Epidemics I were ideal for a high oviposition activity of mosquitoes. It is very difficult to prove if Aedes aegypti or Aedes albopictus was the transmitter mosquito of DF in the epidemic of Thasos Island. To my mind, Aedes aegypti was probably the transmitter mosquito of DF, considering that Aedes albopictus is a recent invader in Europe ${ }^{5}$ and the record of its occurrence in many Regional Units of our country (as Thasos Island which belongs to the Regional Unit of Kavala) the last years, ${ }^{6}$ does not prove its existence in Greece thousands years ago.

\section{Conflict of interest}

None.

\section{References}

1. World Health Organization, Regional Office for South-East Asia. Comprehensive guidelines for prevention and control of dengue and dengue haemorrhagic fever - Revised and expanded edition, 2011. Available at: www.searo.who.int/entity/vector_borne_tropical_ diseases/documents/SEAROTPS60/en/.

2. Kularatne SA. Dengue fever. BMJ 2015;351:h4661.

3.

3. Louis C. Daily newspaper view of dengue fever epidemic, Athens, Greece, 1927-1931. Emerg Infect Dis. 2012;18:78-82.

4. Hippocrates. Epidemics I, 7-8. In: Complete works 13 [in Greek], Athens (Greece): Kaktos Publications, 1993, pp. 49-55.

5. Paupy C, Delatte H, Bagny L, Corbel V, Fontenille D. Aedes albopictus, an arbovirus vector: from the darkness to the light. Microbes Infect. 2009;11(1415):1177-1185.

6. Badieritakis E, Papachristos D, Latinopoulos D, et al. Aedes albopictus (Skuse, 1895) (Diptera: Culicidae) in Greece: 13 years of living with the Asian tiger mosquito. Parasitol Res. 2018;117:453-460. 